\title{
A IMPLANTAÇÃO DA "POLÍTICA DE SAÚDE OCUPACIONAL PARA OS SERVIDORES PÚBLICOS": HISTÓRIAS CONSTRUÍDAS NA UFRJ
}

\author{
The implantation of "Politic of health Occupational from the Publics Servants": \\ histories constructed in UFRJ \\ La implantacin de la "Política de Salúd Ocupacional para los Servidores Públicos": \\ histórias construidas en la UFRJ
}

Washington Ramos Castro

Sheila Nascimento Pereira de Farias

\begin{abstract}
Resumo
0 presente estudo apresenta aspectos relevantes relacionados às construções da saúde do trabalhador na Universidade Federal do Rio de Janeiro (UFRJ), em toda a sua existência. Abordagem especial foi dada aos aspectos da construção que levaram a Divisão de Saúde do Trabalhador da UFRJ a ser uma das entidades parceiras na implementação das "Normas Regulamentadoras de Seguridade Social e de Saúde dos Servidores Públicos Federais Civis no Rio de Janeiro". Trata-se de um levantamento histórico bibliográfico, baseado em fontes primárias e secundárias, que abordou um período de 85 anos da história desta Universidade, em que, inicialmente, não havia um serviço de atendimento à saúde do trabalhador, e também os aspectos políticos de sua criação e desenvolvimento, assim como os desdobramentos práticos e efetivos, decorrentes dos avanços e retrocessos ocorridos no desenvolvimento histórico da atenção à saúde do trabalhador nesta Universidade.
\end{abstract}

Palavras-chave: Saúde Ocupacional. Enfermagem. História. Saúde Pública.

\begin{abstract}
The present presents important aspects to brought into relation with the constructions of worker health on Rio de Janeiro of Federal University (RJFU), during all its existence, aspects of constructions than had realize the Worker Health Division (WHD) of RJFU to be one of member entity in achievement of "Rule Norms of Security Social and of health of Civil Servant Federal Civil on Rio de Janeiro, Brazil". This had special approach It is a historical survey bibliographic, based on primary and secondary documents springs, which have appear 85 years historical lapse of time that university when don't have a service of Worker Health attendee, so how the politics aspects in the time of itself creation and development, so how the practices and effective unroll, them was happened of advance and retrocedely in the process of historical development of Worker Health at attention in this university.
\end{abstract}

Keywords:

Occupational Health. Nurse. History. Public Health.

\section{Resumen}

El presente estudio presenta aspectos relevantes relacionados a las construcciones de la salud del trabajador en la Universidad Federal de Rio de Janeiro (UFRJ), en toda su existencia, ha sido dado abordaje especial a los aspectos de la construcción que llevaron la División de Salud del Trabajador (DVST) de UFRJ a convertirse en una de las entidades colaboradoras en la implantación de las "Normas Reglamentarias de la Seguridad Social y de Salud de los Servidores Públicos Federales Civiles en Rio de Janeiro, Brasil". Se trata de un una pesquisa histórico-bibliográfica, basada en documentos fuentes primarias y secundarias, que han señalado un período de 85 anõs de la historia de esta Universidad en el que no había un servicio de atención a la salud del trabajador, tal como los aspectos políticos de su creación y desarrollo, tal como los desdoblamientos prácticos y efectivos, resultantes de los avances y retrocesos ocurridos en el proceso de desarrollo histórico de atención a la salud del trabajador en esta Universidad.
Palabras clave:

Salud Occupacional. Enfermería. Historia. Salud Pública. 


\section{INTRODUÇÃO}

0 presente estudo remonta a história do Serviço de Perícias e de Saúde Ocupacional da Universidade Federal do Rio de Janeiro (UFRJ), passando pelo surgimento das legislações regulatórias específicas e do Serviço de Saúde Ocupacional nesta Universidade. Assim, traça um paralelo entre os caminhos dos precursores da assistência à saúde do trabalhador nos serviços da Universidade Federal do Rio de Janeiro e a construção das políticas institucionais de atendimento a estes trabalhadores. E como conseqüência, aponta os reflexos decorrentes da aplicação das políticas públicas de atendimento aos trabalhadores das instituições públicas.

Neste sentido, apresenta o desenvolvimento histórico diante dos desdobramentos (modificação das regras periciais) que decorrem da implantação do Sistema Integrado de Saúde Ocupacional dos Servidores Públicos Federais, proposta pelo Ministério do Planejamento, Orçamento e Gestão, em 2005, a partir da aproximação dos modelos propostos, tanto pela Divisão de Saúde do Trabalhador (DVST) da Universidade Federal do Rio de Janeiro, como pela Coordenação de Saúde do Trabalhador da Fundação Oswaldo Cruz (FIOCRUZ).

Poucos estudos se detiveram aos atendimentos de saúde do trabalhador, sob o marco regulatório da Lei 8.112 de 11 de dezembro de 1990, Regime Jurídico Único, e um número reduzido de trabalhos demostram preocupação em avaliar, analisar ou refazer a história de algum serviço médico pericial e de saúde de órgãos públicos. Os que o fazem dão ênfase às análises dos atendimentos, e não ao aspecto histórico. Percebe-se a necessidade de refazer o histórico que levou a Universidade Federal do Rio de Janeiro a ser uma das instituições escolhidas para implementar o Sistema Integrado de Saúde Ocupacional dos Servidores Públicos Federais.

Na vertente pesquisa, o estudo foi relevante por contribuir com a revisão teórica e o levantamento de aspectos históricos que poderão ser subsídio de outros estudos que visem aspectos históricos da Saúde Ocupacional na Universidade Federal do Rio de Janeiro, ao nível de pós-graduação, e, o mesmo tempo, no que se relaciona ao ensino, funcionou como subsídio para o conteúdo teórico que pode ser utilizado nos cursos de graduação.

No que concerne à contribuição para os servidores da Universidade Federal do Rio de Janeiro -UFRJ, permitirá a reflexão e, a partir da compreensão da realidade histórica, a criação de estratégias de Saúde Ocupacional que resultem em melhoria da Qualidade de Vida dos trabalhadores.

\section{OBJ ETIVO}

Analisar aspectos históricos concernentes à implantação da Política de Saúde Ocupacional para os Servidores Públicos Civis das Instituições Públicas Federais do Rio de Janeiro.

\section{METODOLOGIA}

A proposta metodológica do estudo foi de uma pesquisa histórica, cuja análise histórico-documental buscou remontar a história da Saúde Ocupacional da Universidade Federal do Rio de Janeiro, utilizando-se do método indutivo incompleto ou formal, conforme refere Lakatos et al. ${ }^{1}$.

A indução é uma ferramenta que permite se chegar a conclusões que vão além da soma dos dados particulares e dos argumentos originais das premissas.

No presente estudo, foram considerados registros documentais impressos e/ou eletrônicos, pesquisas e discursos de autoridades. Buscou-se pesquisar documentos que explicaram o desenvolvimento do serviço de Saúde Ocupacional na Universidade Federal do Rio de Janeiro. Após a identificação dos documentos, realizou-se uma análise, comparando e organizando as informações, confirmando os fatos.

Segundo Fachin ${ }^{2}$, nas análises documentais, as fontes devem, preferencialmente, ser primárias, uma vez que estes documentos resguardam em sua origem as opiniões ou as bases dos fatos a que se referem.

A abordagem interpretativa para reconstrução histórica norteou os caminhos para identificar os fatos levantados nos documentos que serviram para remontar a história do serviço de saúde ocupacional da Universidade Federal do Rio de Janeiro. Cada informação dos documentos de fontes secundárias que apontou mudança ou aperfeiçoamento das atividades de perícias foi checada e confirmada com base nos documentos (fontes primárias). De acordo com Strauss $^{3}$, ainda que em documentos, o discurso possui elementos possíveis de serem elencados e estudados e é o melhor meio para estudo dos fenômenos sociais a partir de suas manifestações objetivas e subjetivas.

0 cenário deste estudo foi a Universidade Federal do Rio de Janeiro e os locais onde os documentos estavam disponíveis no momento da pesquisa, os locais das reuniões da equipe responsável pela implantação do Convênio: Fundação Oswaldo Cruz/Universidade Federal do Rio de Janeiro, e onde ocorreram eventos ligados ao seu desenvolvimento: o auditório do Ministério do Planejamento, Orçamento e Gestão, na esplanada dos Ministérios em Brasília, a sala de Reunião do Centro de Estudos da Divisão de Saúde do Trabalhador, na llha do Fundão, e as salas 301, 310 e 
314 do Prédio da Escola Nacional de Saúde Pública, da Fundação Oswaldo Cruz, assim como algumas bibliotecas da Universidade Federal do Rio de Janeiro: do Centro de Ciências da Saúde, do Centro de Tecnologia, do Instituto de Filosofia e Ciências Sociais, do Centro de Ciências Matemáticas e da Natureza, e do Centro de Ciências Jurídicas e Econômicas, e o arquivo do Escritório Técnico Universitário.

0 estudo é um levantamento histórico bibliográfico que se desenvolveu de forma seqüencial, obedecendo às etapas científicas necessárias para garantir uma coleta de informações que atenda aos preceitos éticos e técnicos de uma pesquisa histórica. Teve como proposta a reconstrução interpretativa do processo de construção do modelo e do desenvolvimento e da implementação do serviço de perícias médicas e de Saúde Ocupacional da Universidade Federal do Rio de Janeiro.

Fachin ${ }^{2}$ afirma ser através do método histórico que podemos compreender paradigmas e categorias políticas, econômicas, culturais, psicológicas, sociais entre outras. Vislumbrou-se a descrição alcançada a partir da investigação de fatos e acontecimentos ocorridos no passado para a explicação de possíveis projeções destes fatos e sua influência na sociedade contemporânea.

Este método também possibilita analisar a organização das sociedades e das instituições, e sua dinâmica histórica e sua evolução, transformação e desaparecimento. Reconstitui o encadeamento de processos sociais que permitem investigações dos fenômenos (fatos e eventos), dentro de uma perspectiva que possibilita o conhecimento e análise destes fenômenos, de suas causas e de seus efeitos.

Boas, citado por Lakatos et al. ${ }^{4}$, infere que, para compreender a natureza e a função das informações e dos costumes da sociedade contemporânea, deve-se partir do princípio de que as atuais formas de vida social têm origem no passado, sendo importante pesquisar suas raízes.

Acredita-se que o destaque dado a alguns pontos desta história seja apenas uma das possíveis interpretações do próprio processo de busca. Procurou-se descrever aspectos importantes para uma reflexão destas expectativas e frustrações, quanto aos avanços e retrocessos e à ruptura de paradigmas na política de atenção à saúde dos servidores.

\section{APRESENTAÇÃO E DISCUSSÃO DOS RESULTADOS:}

Aspectos relevantes para a compreensão do contexto histórico da saúde do trabalhador na Universidade Federal do Rio de J aneiro:

Da Colônia à República, dezenas de tentativas foram feitas visando à criação de uma universidade pública no Brasil, nenhuma obteve sucesso. De acordo com
Mello ${ }^{5}$, com a chegada da família Real ao Brasil, surgiram cursos destinados a formar profissionais em diversas áreas, também evidenciado em Villanova ${ }^{6}$.

A proclamação da Independência trouxe de volta tal interesse mais eficientemente ${ }^{7}$. A Universidade Federal do Rio de Janeiro, criada em 1920, por decreto presidencial, denominada Universidade do Rio de Janeiro, é considerada a primeira universidade pública brasileira instituída por decreto governamental ${ }^{8}$.

0 primeiro reitor foi o barão de Ramiz Galvão, médico, professor e diretor da Faculdade de Medicina, que permaneceu no cargo até 1925.

0 serviço de atendimento médico de saúde do trabalhador da Universidade Federal do Rio de Janeiro foi instituído em 1968, através de um decreto do Magnífico Reitor Raymundo Augusto de Castro Muniz de Aragão, inicialmente denominado Departamento Médico da Universidade do Brasil, homologado pelo reitor e publicada no Boletim da Universidade do Brasil ${ }^{9}$. Localizou-se, inicialmente, em um dos barracões montados ainda durante o processo de criação da Cidade Universitária. Sendo posteriormente denominada Divisão de Assistência Médica Social, logo recebeu uma sede na Praça da Prefeitura Universitária na llha do Fundão ${ }^{10}$.

Desde a sua criação e, durante 48 de sua existência, a Universidade Federal do Rio de Janeiro não contou com um serviço de Saúde Ocupacional para os seus servidores. Vale ressaltar aqui, para efeito de entendimento histórico, que a falta de legislação específica que servisse de marco regulatório para a montagem de um serviço de saúde próprio determinou a ausência, nessa nova estrutura, de um setor ou serviço que congregasse as atividades de atendimento à saúde ocupacional dos trabalhadores desta universidade; na verdade, as leis eram específicas para cada categoria e havia algumas que ficavam marginalizadas. A Consolidação das Leis do Trabalho (CLT $\left.{ }^{11}\right)$, Lei 5.452, só seria homologada em $1^{\circ}$ de maio de 1943.

Em dezembro de 1985, foi constituída uma comissão para estudar a reestruturação da Divisão de Assistência Médica Social, e, em 2 de abril de 1987, o reitor atribuiu ao vice-reitor a coordenação, direção e execução da reformulação da Divisão de Assistência Médica Social, através da Portaria 332, da Universidade Federal do Rio de Janeiro. Em março de 1988, a nova direção da Divisão de Assistência Médica Social estabelecia prioridades para alcançar os objetivos da reestruturação.

Em setembro de 1989, o diretor da Coordenação de Programas de Pós-Graduação em Engenharia, Professor Luiz Pinguelli Rosa, designando como assessor o Técnico de Segurança do Trabalho da COPPE 
- Centro de Tecnologia, Rubens José Exposito, que atuou de setembro de 1989 até março de 1993 nesta função, desenvolveu projetos em quatro grandes linhas voltadas para a Saúde do Trabalhador: 1) Saúde da Comunidade; 2) Levantamento e Correção dos Ambientes de Trabalho; 3) Reflexão Através do Conhecimento; e 4) Questões Legais ${ }^{12}$.

Neste período, o Congresso Nacional sancionava a Lei 8112, que instituiu o Regime Jurídico Único para os Servidores Públicos Federais. Em 11 de Dezembro de 1990, esta lei foi aprovada e publicada, passando a normatizar as relações trabalhistas dos servidores públicos da União ${ }^{13}$.

A partir de 1991, movimentos dos servidores na Universidade Federal do Rio de Janeiro determinaram muitos avanços na qualidade dos atendimentos pericial e de saúde prestados aos servidores daquela instituição.

A publicação das "Normas para Licenciamento por Motivo de Doença", ocorrida em 12 de março de 1991, e das "Rotinas da Secretaria, Exames Laboratoriais e Licença Médica Fora do Prazo", em 28 de junho de 1995, marcaram um início da regulamentação dos serviços de perícia médica e de serviço de saúde na Universidade Federal do Rio de Janeiro, dentre outras regras que foram propostas e levadas à prática até a publicação do "Estatuto da Seção de Perícias Médicas" e das "Normas e Rotinas da Seção de Perícias Médicas da Universidade Federal do Rio de Janeiro". Muito se caminhou para se chegar ao cenário atual, e estes documentos retirados do Regime Jurídico Único, no período de 1991-1995, nos dão uma noção exata das dimensões de tais mudanças.

Em março de 1992, a Sub-Reitoria de Pessoal da Universidade Federal do Rio de Janeiro, representada pela professora Heloisa Leite, criou um grupo de trabalho, sob a coordenação de Rubens José Exposito, para implantar o Pólo de Saúde do Trabalhador no Centro de Tecnologia. Este projeto não foi efetivado, tendo em vista já existir na Divisão de Assistência Médica Social (DAMES) trabalho semelhante ${ }^{13}$. Em 1993, com nova direção, a DAMES teve seu nome alterado por decisão do Conselho Universitário, passando a se chamar Divisão de Saúde do Trabalhador (DVST). Não se tratou de mera troca de nomes, também foram criadas as Seções de Perícias Médicas, de Engenharia, Segurança e Saúde de Trabalho, e de Programas Especiais.

Logo a seguir, em 1994, foi realizado o "Projeto de Levantamento e Normatização dos Recursos de Assistência à Saúde das Unidades da Universidade Federal do Rio de Janeiro e da Área Programática (A.P
3.1). Foi nomeada uma comissão assessora da SubReitoria de Pessoal, para sistematizar as ações e atividades de assistência à saúde dos trabalhadores da Universidade Federal do Rio de Janeiro e garantir a difusão e a implementação dos preceitos aprovados na II Conferência Nacional de Saúde do Trabalhador, que ocorreu em Brasília em 1994, priorizando as ações de promoção de saúde e prevenção de doenças, e, em junho de 1995, ocorreu o "Projeto de Reestruturação das Atividades do Serviço Social na Divisão de Saúde do Trabalhador" ${ }^{14}$.

Os conceitos contidos na Lei do Sistema Único de Saúde (SUS), Decreto Lei 8.080/90, estiveram no arcabouço destas propostas. Seriam implantados um sistema de informações em Saúde do Trabalhador e três coordenadorias técnicas: 1) de vigilância; 2) de assistência; e 3) de educação, articulados entre si, respondendo a uma coordenadoria geral e a um Conselho de Saúde do Trabalhador da Universidade Federal do Rio de Janeiro ${ }^{13}$.

Reproduziu-se assim exatamente o conceito de hierarquização, com participação popular (controle social) e descentralização dos serviços, previsto no SUS. Toda a estrutura foi baseada na conceituação das Normas Operacionais Básicas, do Sistema Único de Saúde ${ }^{15,16}$.

Em 1998, a Divisão de Saúde do Trabalhador ganha uma nova sede atrás da reitoria, ao lado da garagem, na rua 7 , onde funciona até os dias atuais. Neste período, vários programas de atenção primária à saúde dos trabalhadores da Universidade Federal do Rio de Janeiro foram desenvolvidos (Programas de Atenção ao Trabalhador): DST/AIDS no local de trabalho; Alcoolismo; Saúde Mental do Trabalhador; Diabetes; Hipertensão Arterial; Nutrição/Obesidade; Saúde Oral; Psicodrama e Tabagismo.

Vale ressaltar que, a partir da publicação do Regime Jurídico Único, após avaliação e anuência do Conselho Universitário e a aprovação por aquele fórum, a Divisão de Saúde do Trabalhador passou a responder pelas atividades de perícias médicas e de saúde, inclusive àquelas que se estendiam por mais de 15 dias. A Divisão de Saúde do Trabalhador passou a dar licenças desde 0 início dos afastamentos até o momento da aposentadoria, quando fosse 0 caso, após 730 dias de licença ${ }^{10}$.

Em dezembro de 2002, foi realizado o I Seminário Interno da Divisão de Saúde do Trabalhador com o objetivo de reorganizar os serviços oferecidos e apresentar novas propostas de trabalho, reordenando a Divisão ${ }^{17}$.

Por iniciativa da Pró-Reitoria de Pessoal (PR-4), realizou-se um encontro em julho de 2003 , onde 
estavam presentes diversos dos responsáveis pelos serviços de Recursos Humanos e dos Departamentos de Pessoal das Unidades e Centros da Universidade Federal do Rio de Janeiro, além de muitas Unidades Acadêmicas e do Sindicato dos Trabalhadores da Universidade Federal do Rio de Janeiro (SINTUFRJ), que determinaram, entre outras coisas, a formação de um grupo de trabalho para ampliar a discussão sobre a "Política de Saúde dos Servidores da Universidade Federal do Rio de Janeiro" e que foram incumbidos também de organizar um evento para discutir a situação das políticas públicas e institucionais, referentes à saúde dos trabalhadores da Universidade Federal do Rio de Janeiro.

A partir deste evento, que foi intitulado I Seminário de Saúde da PR-4 ${ }^{17}$, publicado nos anais do I Congresso Interno da PR-4 em 2003, houve uma aproximação desta Pró-Reitoria com as Unidades da Universidade Federal do Rio de Janeiro, em especial com a Divisão de Saúde do Trabalhador. Questões de pessoal foram levadas para o Conselho Universitário, e houve investimento na capacitação dos servidores; desenvolveu-se uma política de capacitação, conforme demandas das Unidades; calendários bimestrais de cursos de pequena duração e eventos foram elaborados, e alterou-se a política de saúde dos servidores, adequando-a às Normas Regulamentadoras ${ }^{18}$, pacificadas pela Lei 6.367 de 19 de outubro de 1976.

Exames periódicos vêm sendo retomados pela Divisão de Saúde do Trabalhador, e trabalhou-se com programas preventivos, desenvolvidos por esta Divisão. Também se conseguiu levar ao Ministério de Educação (MEC) e ao Ministério do Planejamento, Orçamento e Gestão a deficiência numérica de pessoal existente no dimensionamento do quadro geral de servidores desta Universidade.

A Divisão de Saúde do Trabalhador vem ampliando suas atividades e atribuições. Paralelamente, outras iniciativas isoladas vêm sendo desenvolvidas na Universidade Federal do Rio de Janeiro, tais como: Centro de Ciências da Saúde; o projeto Segurança Ambiental e Biossegurança (SABIOS I), sob a coordenação técnica da Escola Politécnica/CT; o Projeto Pró-Saúde do Hospital Escola São Francisco de Assis, visando identificar riscos à saúde dos funcionários e propor atividades preventivas e promotoras de saúde; a Comissão de Biossegurança do Centro de Ciências da Saúde, segurança e qualidade de vida dentro do Centro; o Grupo de
Gestão Tecnológica - Programa de Gestão Ambiental do Instituto de Macromoléculas; a Estação Experimental de Tratamento de Esgoto da Cidade Universitária/Escola Politécnica; o Serviço de Saúde do Trabalhador do Hospital Universitário Clementino Fraga Filho/Seção de Segurança e Saúde do Trabalhador, estrutura que se tornou um dos pólos de descentralização da Divisão de Saúde do Trabalhador e que atualmente está em dificuldades operacionais por falta de pessoal; e a Central de Emergência do Centro de Tecnologia/Coordenação de Programas de Pós-Graduação em Engenharia ${ }^{10}$.

No II Seminário, em dezembro de 2003, discutiuse a implantação das resoluções do I Seminário. Observou-se que, após o III Seminário Interno da Divisão de Saúde do Trabalhador, em dezembro de 2004, houve um momento de grande mobilização dos servidores daquela Divisão, que passava por reformulação de sua estrutura interna e também realizava a implantação do Programa de Qualidade do Serviço Público (PQSP).

Logo após a realização deste seminário, que contou com a presença do coordenador de recursos humanos do Ministério do Planejamento, Orçamento e Gestão Luiz Rober to Pires Domingues Junior, houve um contato do Ministério do Planejamento, Orçamento e Gestão para apresentar uma proposta interessante, para mudar a estrutura de atenção à saúde do trabalhador, através da implementação do Sistema Integrado de Saúde Ocupacional dos Servidores Públicos Federais.

A estrutura proposta pela Norma Regulamentadora apresentada pelo Ministério do Planejamento, Orçamento e Gestão foi concebida e organizada de forma a permitir a ação de um Controle social, representada pelo direito a voz e voto dos próprios servidores, organizados pelas suas representações classistas.

Segundo Castro $^{10}$, uma comissão com representação dos trabalhadores e do governo, assim como das instituições envolvidas na consecução desta proposta, foi criada nacionalmente e implantada antes que se desse por finalizado o Sistema Integrado de Saúde Ocupacional dos Servidores Públicos Federais. Esta comissão passou a se reunir mensalmente em Brasília, pretendendo ser um fórum deliberativo, o que dificultou muito as negociações, em virtude da postura tomada por algumas das representações.

Nesta comissão tiveram assento todas as representações centrais dos servidores públicos federais, além das entidades envolvidas nos modelos 
experimentais das quatro capitais e do Governo Federal através do Ministério do Planejamento, Orçamento e Gestão, do Ministério do Trabalho e Emprego, do Instituto Nacional de Seguridade Social e do Ministério da Saúde, buscando construir mecanismos que subsidiem a saúde do trabalhador.

No que se refere aos aspectos históricos-jurídicos, evidenciam-se alguns eventos que merecem destaque.

A partir da Carta Imperial de 1824, até a Constituição Federal $\left(\mathrm{CF}^{19}\right)$ de 1988 , sempre houve referências aos princípios basilares do direito à saúde e à segurança no meio ambiente do trabalho ${ }^{13}$. Quanto aos direitos dos trabalhadores, a Constituição Federal, artigo $7^{\circ}$, Inciso XXII, estabelece a redução dos riscos inerentes ao trabalho por meio de normas de saúde, higiene e segurança.

Assim, reforçando esse entendimento, o Regime Jurídico Único, em seu artigo 230, refere que a assistência à saúde do servidor, ativo ou inativo, e de sua família, compreende assistência médica, hospitalar, odontológica, psicológica e farmacêutica, prestada pelo Sistema Único de Saúde (SUS) ou diretamente pelo órgão ou entidade ao qual estiver vinculado o servidor, ou, ainda, mediante convênio ou contrato na forma estabelecida em regulamento.

De acordo com as disposições gerais da Lei 8.080 de 1990, do Sistema Único de Saúde (SUS), em suas disposições gerais, indica-se que o dever do Estado não exclui o das pessoas, da família, das empresas e da sociedade. Verifica-se, ainda, como princípios e diretrizes, que as ações e serviços públicos de saúde e os serviços privados contratados ou conveniados que integram SUS sejam desenvolvidos de acordo com as diretrizes previstas no Art. 198 da Constituição Federal.

Assim, destacam-se alguns princípios utilizados nas ações de saúde: I) Universalidade de acesso aos serviços de saúde em todos os níveis de assistência; II) Integralidade de assistência, entendida como conjunto articulado e contínuo das ações e serviços preventivos e curativos, individuais e coletivos, exigidos para cada caso em todos os níveis de complexidade do sistema; III) Preservação da autonomia das pessoas na defesa de sua integridade física e moral; IV) Igualdade da assistência à saúde, sem preconceitos ou privilégios de qualquer espécie; V) Direito à informação, às pessoas assistidas, sobre sua saúde; VI) Divulgação de informações quanto ao potencial dos serviços de saúde e a sua utilização pelo usuário; VII) Utilização da epidemiologia para o estabelecimento de prioridades, a alocação de recursos e a orientação programática; VIII)
Participação da comunidade; IX) Regionalização e hierarquização da rede de serviços de saúde; X) Integração em nível executivo das ações de saúde, meio ambiente e saneamento básico; XI) Capacidade de resolução dos serviços em todos os níveis de assistência; e XII) Organização dos serviços públicos de modo a evitar duplicidade de meios para fins idênticos.

Portanto, de acordo com o exposto, verificou-se a proposição do documento "Política de Saúde Integral do Corpo Social da Universidade Federal do Rio de Janeiro", que foi um marco importante no desenvolvimento de políticas afirmativas na área de saúde do trabalhador. 0 documento foi elaborado a partir das discussões dos grupos e subgrupos que se formaram pelos desdobramentos das ações propostas no Seminário de saúde da Universidade Federal do Rio de Janeiro, em 2003, patrocinado pela Reitoria e realizado pela Pró-Reitoria de pessoal (PR-4), em consonância com a Divisão de Saúde do Trabalhador.

0 documento em tela constitui-se em um conjunto de preceitos legais que visa garantir a gerência integrada das ações de segurança do trabalho, saneamento do meio ambiente e acesso à saúde, garantindo a qualidade de vida do corpo social da Universidade Federal do Rio de Janeiro ${ }^{18}$. 0 desenvolvimento das ações propostas ocorreu sob 0 comando da Divisão de Saúde do Trabalhador, com o apoio institucional da PR-4.

Trata-se, portanto, de um importante marco conceitual regulatório, produto de ações pragmáticas da estrutura de saúde em parceria com os próprios trabalhadores da Universidade Federal do Rio de Janeiro, em sua breve história de vida ${ }^{18}$.

Há muitos pontos em comum entre o documento criado na Universidade Federal do Rio de Janeiro e o gerado no Ministério do Planejamento, Orçamento e Gestão, que se intitula "Norma Regulamentadora", a qual pretende preencher as lacunas existentes na legislação específica do serviço público. Provavelmente, as ações determinadas pelo I Seminário tenham influenciado na escolha da Universidade Federal do Rio de Janeiro como uma das instituições do Rio de Janeiro, que hoje é responsável pela implementação do "Sistema Integrado de Saúde Ocupacional dos Servidores Públicos Federais", através do convênio firmado entre a Universidade Federal do Rio de Janeiro e a Fundação Oswaldo Cruz.

Estas são vitórias das mobilizações dos trabalhadores na construção da política de saúde da Universidade Federal do Rio de Janeiro e que não 
podem deixar de ser vistas como modelo, ou como as bases para criação de um modelo, ideal para o cuidado com a Saúde Ocupacional de servidores públicos.

0 documento "Política de Saúde Integral do Corpo Social da Universidade Federal do Rio de Janeiro" apresenta proposições que guardam uma aproximação com a Lei 8.080, de 19 de setembro de 1990, e com as suas Normas Operacionais Básicas: $1 / 96$ de 5 de novembro de 1996 e 1/98 de 2 de janeiro de $1998^{15,16}$.

Nesse documento, encontramos a proposição da instituição das Comissões Locais de Saúde do Trabalhador, que se tornaram a estrutura para os trabalhadores regidos pelo Regime Jurídico Único (RJU), e da Comissão Interna de Prevenção de Acidentes (CIPA).

\section{CONCLUSÃO}

A ausência de legislação regulamentadora dos direitos à saúde do trabalhador para os funcionários civis do serviço público federal não foi alterada de forma significativa com a promulgação do Regime Jurídico Único, existindo uma lacuna quanto à promoção da saúde e à prevenção de riscos, especialmente relacionada aos riscos ocupacionais e de acidentes de trabalho. A proposta feita pelo Ministério do

\section{Referências}

1. Lakatos EM, Marconi MA. Metodologia do trabalho científico. $3^{\mathrm{a}}$ ed. São Paulo (SP): Atlas; 1991.

2. Fachin O. Fundamentos de metodologia, São Paulo (SP): Atlas; 1993.

3. Strauss CL. Antropologia estrutural, Rio de Janeiro(RJ): Tempo Brasileiro; 1975.

4. Lakatos EM, Marconi MA. Fundamentos de metodologia científica. $7^{\text {a }}$ ed. São Paulo (SP): Atlas; 1998.

5. Mello JR, ACM. Hospitais brasileiros: histórias diferentes, realidades iguais. Resumos dos trabalhos apresentados no Congresso Brasileiro de Estudantes de Medicina; 1985 jul. 13-18; Fortaleza (CE), Brasil. Fortaleza(CE): UECE; 1985. p. 13.

6. Villanova JBA. Arquitetura moderna em debate. Rev Teoria e Debate 1948 jul./dez; 1(11): 23- 4.

7. Lobo TBA. Universidade Pública no Brasil. [tese de mestrado]. Niterói (RJ): Faculdade de História/ UFF; 1980.

8. Fávero PA.A hstória concisa da saúde no Distrito Federal da Guanabara.Revisão histórica [tese de doutorado]. Rio de Janeiro (RJ):Faculdade de História da Universidade do Brasil; 1977.
Planejamento, Orçamento e Gestão pretende resolver esta lacuna promulgando a Norma Regulamentadora de Saúde e Seguridade Social dos Servidores Públicos Civis de Órgãos Públicos Federais.

A discussão iniciada com a publicação do DecretoLei 8.112 em 11 de dezembro de 1990 está sendo retornada, tendo em vista as lacunas no que concerne aos direitos dos trabalhadores, principalmente nos aspectos existentes em algumas normas regulamentadoras da CLT da área privada, que atualmente são usadas por analogia para os Servidores Públicos, tais como o Programa de Prevenção de Riscos e acidentes (PPRA), o Programa de Controle Médico em Saúde Ocupacional (PCMSO), o Perfil Profissiográfico Previdenciário (PPP) e a Comissão Interna de Prevenção de Acidentes (CIPA).

Destaca-se, portanto, a importância da criação de uma norma específica para os trabalhadores de órgãos públicos federais, ressaltando — se, ainda, a necessidade de ampla discussão com os trabalhadores, que resulte em melhor qualidade de vida no trabalho dos servidores de instituições públicas, por meio da aplicação dos preceitos constitucionais, expostos na Constituição Federal ${ }^{19}$.

9. Universidade Federal do Rio de Janeiro. Boletim da Universidade do Brasil 1968 out/dez.

10. Castro WR. Coluna reconhecimento e recompensa. Informe SEPE. [periódico on line] 2004 jan; [citado 25 maio 2005]: 1 (2): [aprox.4 telas]. Disponível em: http://www.ufrj.br/pr4/publicacoes/ informesepea1n1.html

11. Lei $n^{0} 5452$ de $1^{\circ}$ de maio de 1943. Cria a Consolidação das Leis do Trabalho-CLT e da outras providências. Diário Oficial da República Federativa do Brasil, Brasília (DF), 20 maio 1943. Seção 1: 1.

12. Universidade Federal do Rio de Janeiro. Divisão de Saúde do Trabalhador. Relatório do $1^{\circ}$ Seminário Interno da Divisão de Saúde do Trabalhador. Documento final. Rio de Janeiro (RJ); 2004 dez. Documento impresso.

13. Moraes JC. Políticas de saúde e estratégias de organização dos serviços públicos na cidade do Rio de Janeiro. Relatório Técnico. Secretaria de Estado de Saúde. Rio de Janeiro (RJ); 2002.

14. Universidade Federal do Rio de Janeiro. Divisão de Saúde do Trabalhador. Memorando/Circular DVST n ${ }^{\circ} 433$ de 26 de novembro de 1994. Projeto de levantamento e normatização dos recursos de assistência à saúde das unidades da Universidade Federal do Rio de Janeiro e da Área Programática 3.1; 1994.

15. Ministério da Saúde (BR). Norma Operacional Básica do Sistema Único de Saúde - NOB/SUS n01 / 1996. Brasília(DF); 1996. 
16. Ministério da Saúde (BR). Norma Operacional Básica do Sistema Único de Saúde- NOB/SUS n ¹/1998. Brasília(DF); 1998.

17. Universidade Federal do Rio de Janeiro. Anais do $1^{\circ}$ Congresso Interno da PR-4; 2003. Rio de Janeiro (RJ); 2004. Documento impresso.

18. Ministério do Trabalho(BR). Portaria MT no 3.214 de 08 de junho de 1978 , Passifica as normas regulamentadoras $\mathrm{n}^{0} 1$ a 32 previstas no capítulo $5^{\circ}$ da CLT. Diário Oficial da República Federativa do Brasil, Brasília (DF), 08 jun 1978. Seção 1: 1.

19. Constituição Federal da República Brasileira. Senado Federal. 1988. Diário Oficial da República Federativa do Brasil, Brasília (DF), 20 maio 1988. Seção 1: 1.

\section{Sobre as Autoras}

\section{Washington Ramos Castro}

Enfermeiro Especialista em Enfermagem do Trabalho, Especialista em atendimento à catástrofes com múltiplas vítimas e Especialista em Saúde da Família. - Assessor da Direção do Instituto de Ginecologia da UFRJ - washignton@ufrj.br

\section{Sheila Nascimento Pereira de Farias}

Professora e Doutora do Departamento de Enfermagem em Saúde Pública da Escola de Enfermagem Anna Nery/ UFRJ. 